\title{
Acute effects of one session dry needling on a chronic golfer's elbow disability
}

\author{
Ardalan Shariat ${ }^{1}$, Pardis Noormohammadpour, ${ }^{1, *}$, Amir Hossein Memari', Noureddin Nakhostin Ansari ${ }^{1,2}$, Joshua A. Cleland ${ }^{3}$, \\ Ramin Kordi'
}

'Sports Medicine Research Center, Neuroscience Institute, Tehran University of Medical Sciences, Tehran, Iran 2Department of Physiotherapy, School of Rehabilitation, Tehran University of Medical Sciences, Tehran, Iran

${ }^{3}$ Franklin Pierce University, Rindge, NH, USA

A 40-year-old retired male elite athlete with more than 20 years' experience in wrestling and bodybuilding with mild elbow pain and disability of 2 years presented to our clinic reporting an acute pain in medial aspect of the elbow. Physical examination revealed symptoms of left Golfer's elbow during target-directed movements. The results of sonography in left elbow showed low level of hypo echo irregularity and increased blood flow in color Doppler mode in the common flexor origin. The pain amplitude was moderate at rest and extremely high during kinetic and intentional movements. During last 2 years, the patient tried different types of therapy including manual therapy, Transcutaneous electrical nerve stimulation and also received a variety of injections none of which were effective for reducing pain and disability. This is the first study showed that one sessions of dry needling improved both pain and disability and helped to return to a normal life and exercise training.

Keywords: Movement disorders, Ultrasonography, Disability evaluation, Doppler, Exercise

\section{INTRODUCTION}

Physical therapy is a conservative management approach for the treatment of pain, disability and maximizing satisfaction in patients with lateral epicondylitis (Sevier and Wilson, 1999). Using modalities such as heat, dry needling, ultrasound and transcutaneous electrical nerve stimulation (TENS) has been recommended as an effective treatment for this population (Gattie et al., 2017). These treatments have been purported to increase soft tissue extensibility and blood flow along with reducing pain and muscle spasms (Sevier and Wilson, 1999).

Dry needling is a technique in which a fine sterile needle is used to penetrate the skin, subcutaneous tissues, and muscle, with the intent to mechanically affect soft tissue without the use of an anesthetic (Venâncio et al., 2008). It is a common treatment technique in orthopaedic manual physical therapy (Dommerholt,
2011) and is often used to treat myofascial trigger points, which are described as localized hypersensitive spots in a palpable taut band of muscles (Geist et al., 2017). Dry needling might not change some central sensitization aspects, also it is probable that referred and local pain is reduced, range of motion and patterns of muscle activation is improved and trigger points' of chemical environment is changed (Dommerholt, 2011). Randomized controlled studies have failed to demonstrate meaningful effects of dry needling for chronic elbow disability. Despite the availability of other strategies for continuous therapies that provide reasonable management of chronic elbow disabilities, some therapists continue to use intensive dry needling for long duration (Shanmugam et al., 2015). Local effects of dry needling have shown significant pain relief in musculoskeletal syndromes of the shoulder or elbow, but no study has evaluated the effect of dry needling on chronic Golfer's elbow syndrome in a retired experienced athlete. We de-
${ }^{*}$ Corresponding author: Pardis Noormohammadpour

(iD) https://orcid.org/0000-0001-5153-9565

Sports Medicine Research Center, Neuroscience Institute, Tehran University of

Medical Sciences, Tehran 14395-578, Iran

Tel: +98-21-8863-0227, Fax: +98-21-8800-3539

E-mail: normohamadpour@tums.ac.ir

Received: January 7, 2018 / Accepted: January 29, 2018
This is an Open Access article distributed under the terms of the Creative Commons Attribution Non-Commercial License (http://creativecommons.org/licenses/by-nc/4.0/) which permits unrestricted non-commercial use, distribution, and reproduction in any medium, provided the original work is properly cited. 
scribe a case where this treatment was performed on the elbow flexors in one session of dry needling therapy. This study was carried out according to the standard reported in the Declaration of Helsinki and the patient's consent was obtained by the patient signing the terms of consent.

\section{MATERIALS AND METHODS}

\section{Case history}

The approval of the Research Ethics Committee of the Sports Medicine Research Center was obtained on their monthly meeting on 15 October 2017 (approval number: IR.TUMS.REC. 2871). A 40-year-old retired, male, elite, athlete with more than 20 years' experience in wrestling and bodybuilding presented with mild elbow disability (based on the results of physical examination done by a Sports Medicine specialist) 2 years duration and a 2 months worsening of symptoms. His medical history included chronic lower back pain, scoliosis, and hypercholesterolemia. $\mathrm{He}$ had retired from professional training 3 years ago, and 2 years ago he started training with Thera-band and elastic rope. Then, symptoms of Golfer's elbow slowly appeared and was followed by a mild disability in his left elbow, however, the results of magnetic resonance imaging (MRI) at that time were normal. This refers to his disability in performing daily activities with high forces on the elbow such as carrying a heavy object, or doing heavy household chores.

During the last year he visited a chiropractic specialist, physiotherapist and, an orthopedic expert. During his visits to other health care professionals he received around 9 sessions of manual therapy (deep friction massage and Graston massage), around 12 sessions of physiotherapy and TENS, and injected 2 times with dexamethasone $4 \mathrm{mg} / \mathrm{mL}$ and used Ibuprofen $800 \mathrm{mg}$ (3 times a day for 2 weeks). He injected triamcinolone acetonide $40 \mathrm{mg}, 2 \%$ lidocaine $2 \mathrm{~mL}$ at the area of pain. He attended hydrotherapy treatments (range of motion exercises in water for the shoulder and elbow) 3 times per week for 3 weeks with each session lasting 20-35 min. He received 5 sessions dry needling to the shoulder and hand area as every 3 days for 15 days. He was also treated with Graston Technique on his triceps area in reverse direction. He also used acupotomy (small needle-scalpel therapy) for 4 sessions on the medial side of his left elbow.

\section{Evaluation}

There is a paucity of literature on different protocols of dry needling treatment for concurrent symptoms of Golfer's elbow espe- cially for chronic injuries. So, the subjective measures of this study were based on observing a localized tenderness just below the medial epicondyle with pain, and having a limitation in forceful wrist flexion and a limitation in forearm pronation against resistance. Once he was referred to our clinic he had severe pain and when we asked him to rate his pain based on visual analogue score from 0 (no pain) to 10 (the worst pain that he can imagine) he selected 7. We examined movements in the hand, shoulder and elbow area, based on a standardized method for assessment of elbow function, introduced in 1999 by the American Shoulder and Elbow Surgeons committee (King et al., 1999). We also examined his elbow area through sonography. His movement in the left elbow was painful and he was anxious. He also reported serious physical disability occurred after his first injection around 8 months ago. The result of sonography demonstrated a minor amount of inflammation around his elbow, but it was not significant (Fig. 1). It should be mentioned that 3 months before coming to our center he was not using any anti-inflammatory drugs and he was not using any treatment and only had daily exercise of walking on Treadmill with intensity around $60 \%-70 \%$ of maximum oxygen uptake.

The objective measure of this study was based on the appearance of a focal hypoechoic area and increasing color Doppler flow in the place of maximum tenderness, which could support for medial epicondyle tendinosis. The main aim of this therapy was to improve the elbow disability by decreasing the level of pain in his daily tasks and duties in a normal life including driving, washing, etc.

\section{Intervention}

For the deep dry needling treatment, we asked him to lay down on the bed. A Sports Medicine Specialist, put a pillow under his hand and after cleaning the zone with alcohol. She used 10 stainless steel dry needles (size, $0.25 \mathrm{~mm} \times 25 \mathrm{~mm}$; SMC, Seoul, Korea) on his flexor area (medial epicondyle) and inner side of left elbow which she kept in place for $20 \mathrm{~min}$ (Fig. 1). The needles were placed vertically inside the muscle for a height around $0.5-1 \mathrm{~cm}$. The distance between needles was $0.5-1.5 \mathrm{~cm}$ (Fig. 2).

\section{RESULTS}

After the dry needling, we placed an ice pack around the area to reduce the soreness that may be associated with dry needling. There were no changes in his range of motion and pain immediately after dry needling. The patient returned for a follow-up two 

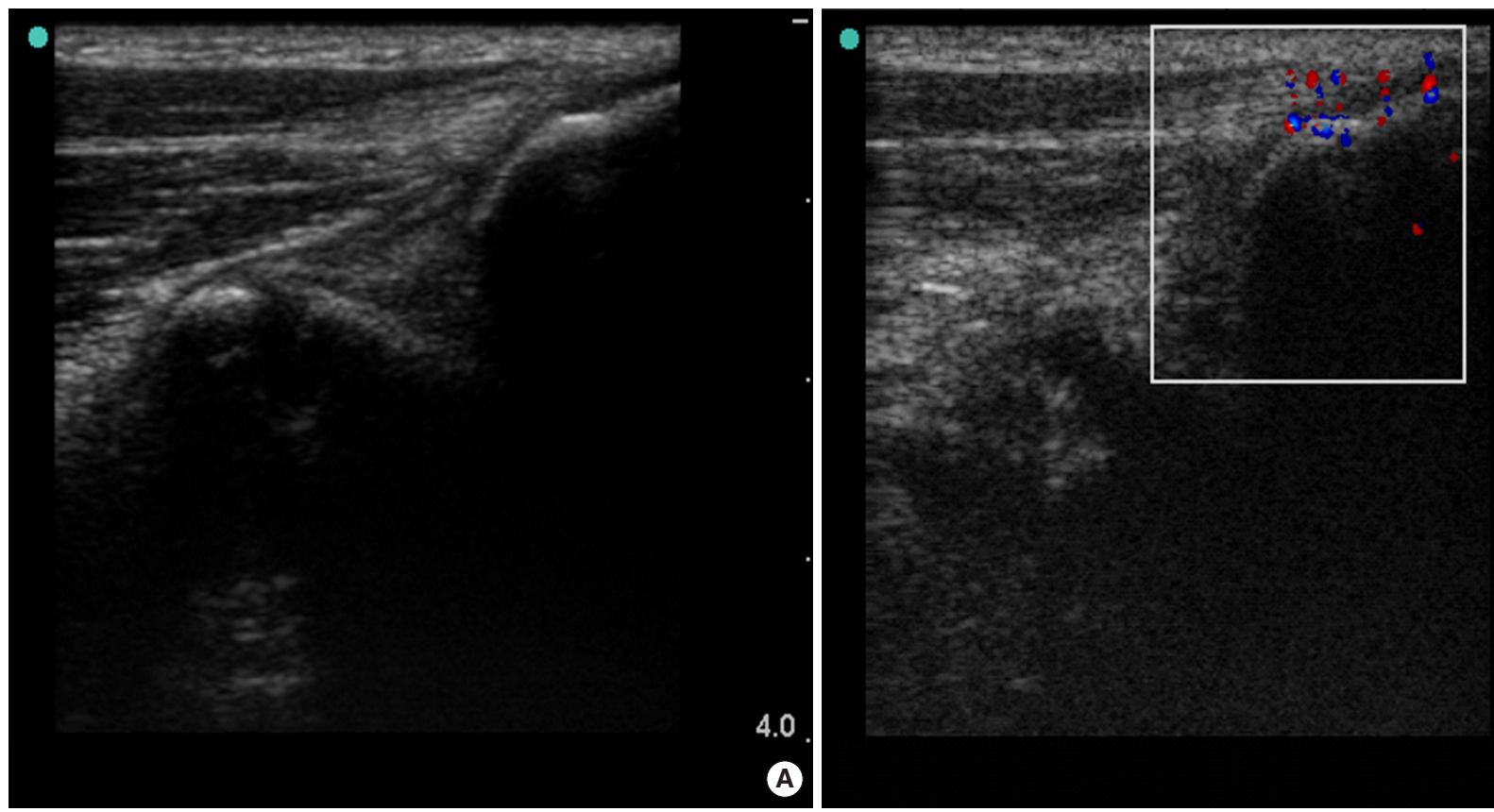

Fig. 1. Result of sonography. Medial elbow tendinopathy and insertion of common flexor origin at medial epicondyle, in a longitudinal view: (A) Grayscale B-mode ultrasound shows irregularity and a focal hypoechoic area in the common flexor origin, (B) Color Doppler ultrasound shows increased blood flow inside the hypoechoic area. The red color dots show the blood flow towards the transducer, and blue ones show the blood flow away from the transducer.

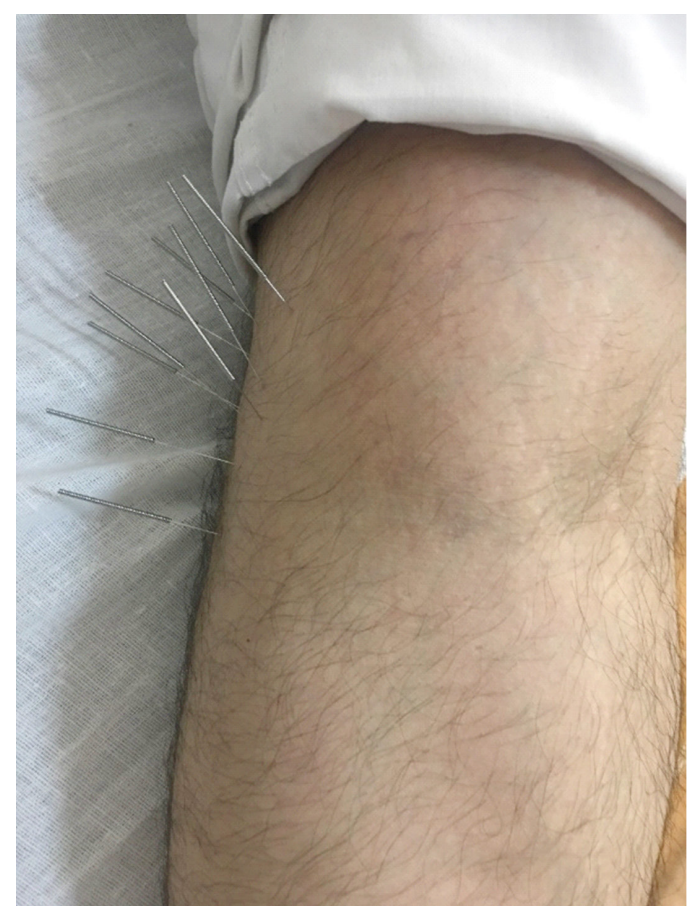

Fig. 2. Dry needling on medial epicondyle.

days after the initial therapy session. He reported less pain and was able to do more activities functionally associated with the elbow. His movements were improved and there was no specific movement limitation. Seven days after his therapy session, he started his exercise training with elastic bands pain free.

\section{DISCUSSION}

The diagnosis of Golfer's elbow is essentially clinically based on a good observation and physical examination (Zeisig and Fahlström, 2015). It can be misdiagnosed with medial collateral ligament injury, valgus instability, cervical spine radiculopathy, ulnar nerve entrapment in the grove (ulnar neuritis) (Barco and Antuña, 2017). Most of the time patients have a normal MRI/x-ray report. Although the cause of Golfer's elbow is unknown it appears to be associated with a combination of mechanical overloading and abnormal microvascular responses which have been found at the site of tendinopathy (Sung et al., 2016).

Acute dry needling, especially on the elbow area is rare and previous studies that have used dry needling have included multiple sessions and mostly on those with acute pain/disability. In our case, the therapy was performed in one session only. The result of this case study showed that applying dry needling to the pain sources for one session only may be helpful in improving Golfer's elbow pain and disability.

There is not much study about the therapeutic effects of dry needling on lateral epicondylitis especially among athletes/retired 
athletes, but in 2013, Stenhouse et al. (2013) showed the positive effects of acupuncture and dry needling therapy for refractory of lateral epicondylitis. After it in 2014, Mishra et al. (2014), showed the positive effect of dry needling with platelet-rich plasma in comparison with dry needling alone on lateral epicondylitis. In addition, there was an interesting study done by González-Iglesias et al. (2011), and suggested dry needling as an effective method for management of lateral epicondylalgia in rock climbers.

To our knowledge, this is the first time that a chronic Golfer's elbow in a retired athlete with increasing blood flow in color Doppler on sonography was treated by one session of dry needling. However, we believe that there exists many gaps in the literature and future studies should establish the accurate placement of dry needling and define the necessary number of sessions for optimal therapy. In addition, we are not sure if this type of acute dry needling could be useful for other parts of body.

We believe that, if the needling be done in the proper location it might be useful and the amount of medications used could potentially be reduced. Furthermore, the intensive needling can irritate the soft tissue and might directly impact the level of pain. This case illustrates an approach in the administration of dry needling that can potentially result in a rare and long forgotten adverse outcome of the treatment which can cause significant patient pain and movement disability.

In conclusion, joint disability is a very common injury among athletes and individuals who have the potential to develop overuse syndromes. Clinicians should be aware of this possibility and devote attention to the physical therapies without side effects and low cost for the patients. Otherwise, joint injuries can easily be misdiagnosed, leading to the use of an unnecessary, inappropriate or even dangerous medication or therapy.

As the physical performance of the patient was improved after two days, and he reported less pain, we did not use sonography for the second time. However, it would be interesting if future studies use sonography procedure in follow up tests and compare the before and after sonographies.

\section{CONFLICT OF INTEREST}

No potential conflict of interest relevant to this article was reported.

\section{ACKNOWLEDGMENTS}

Our special thanks go to Mr. Majid Garak Yaragh, who accept- ed to be as our patient and let us to publish his elbow and sonography pictures.

\section{REFERENCES}

Barco R, Antuña SA. Medial elbow pain. EFORT Open Rev 2017;2:362371.

Dommerholt J. Dry needling - peripheral and central considerations. J Man Manip Ther 2011;19:223-227.

Gattie E, Cleland JA, Snodgrass S. The effectiveness of trigger point dry needling for musculoskeletal conditions by physical therapists: a systematic review and meta-analysis. J Orthop Sports Phys Ther 2017;47: 133-149.

Geist K, Bradley C, Hofman A, Koester R, Roche F, Shields A, Frierson E, Rossi A, Johanson M. Clinical effects of dry needling among asymptomatic individuals with hamstring tightness: a randomized controlled trial. J Sport Rehabil 2017;26:507-517.

González-Iglesias J, Cleland JA, del Rosario Gutierrez-Vega M, Fernández-de-las-Peñas C. Multimodal management of lateral epicondylalgia in rock climbers: a prospective case series. J Manipulative Physiol Ther 2011;34:635-642.

King GJ, Richards RR, Zuckerman JD, Blasier R, Dillman C, Friedman RJ, Gartsman GM, Iannotti JP, Murnahan JP, Mow VC, Woo SL. A standardized method for assessment of elbow function. Research Committee, American Shoulder and Elbow Surgeons. J Shoulder Elbow Surg 1999;8:351-354.

Mishra AK, Skrepnik NV, Edwards SG, Jones GL, Sampson S, Vermillion DA, Ramsey ML, Karli DC, Rettig AC. Efficacy of platelet-rich plasma for chronic tennis elbow: a double-blind, prospective, multicenter, randomized controlled trial of 230 patients. Am J Sports Med 2014;42: 463-471.

Sevier TL, Wilson JK. Treating lateral epicondylitis. Sports Med 1999;28: 375-380.

Shanmugam S, Mathias L, Rai S. Early effects of dry needling and low level laser therapy in chronic tennis elbow-an experimental study. Int J Health Sci Res 2015;5:187-196.

Stenhouse G, Sookur P, Watson M. Do blood growth factors offer additional benefit in refractory lateral epicondylitis? A prospective, randomized pilot trial of dry needling as a stand-alone procedure versus dry needling and autologous conditioned plasma. Skeletal Radiol 2013;42:1515-1520.

Sung DJ, Park SJ, Kim S, Kwon MS, Lim YT. Effects of core and non-dominant arm strength training on drive distance in elite golfers. J Sport Health Sci 2016;5:219-225.

Zeisig E, Fahlström M. Lateral and medial elbow tendinopathies. In: Doral 
MN, Karlsson J, editors. Sports injuries: prevention, diagnosis, treatment and rehabilitation; 2015. p. 587-592.

Venâncio Rde A, Alencar FG, Zamperini C. Different substances and dry- needling injections in patients with myofascial pain and headaches. Cranio 2008;26:96-103. 